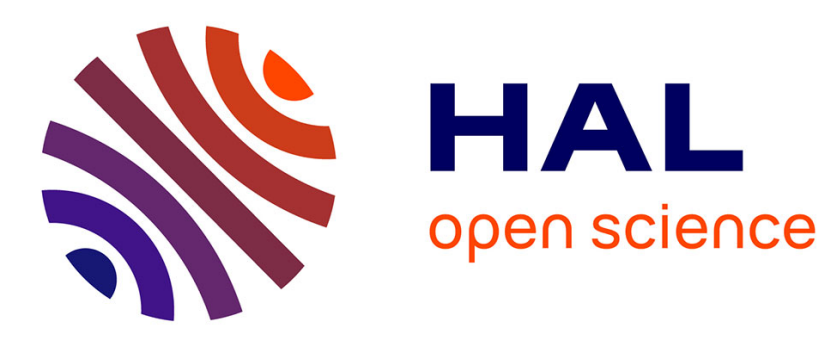

\title{
Bubble and Hermite Natural Element Approximations
}

Julien Yvonnet, Pierre Villon, Francisco Chinesta

\section{To cite this version:}

Julien Yvonnet, Pierre Villon, Francisco Chinesta. Bubble and Hermite Natural Element Approximations. Lecture Notes on Computational Science and Engineering, pp.283-298, 2007, 10.1007/978-3540-46222-4_17. hal-01007481

\section{HAL Id: hal-01007481 \\ https://hal.science/hal-01007481}

Submitted on 9 Oct 2017

HAL is a multi-disciplinary open access archive for the deposit and dissemination of scientific research documents, whether they are published or not. The documents may come from teaching and research institutions in France or abroad, or from public or private research centers.
L'archive ouverte pluridisciplinaire HAL, est destinée au dépôt et à la diffusion de documents scientifiques de niveau recherche, publiés ou non, émanant des établissements d'enseignement et de recherche français ou étrangers, des laboratoires publics ou privés. 


\title{
Bubble and Hermite Natural Element Approximations
}

\author{
J. Yvonnet ${ }^{1}$, P. Villon ${ }^{2}$, and F. Chinesta ${ }^{3}$ \\ 1 Université de Marne-la-Vallée, 5 Bd Descartes, 77454 Marne-la-Vallée Cedex, \\ France \\ jyvonnet@univ-mlv.fr \\ 2 Université de Technlogies de Compiègne, BP 20529, 60205 Compiègne cedex, \\ France \\ pierre.villon@utc.fr \\ 3 École Nationale Supérieure d'Arts et Métiers, 151 boulevard de l'Hôpital, \\ F-75013 Paris, France \\ francisco.chinesta@paris.ensam.fr
}

Summary. In this paper, new natural element approximations are proposed, in order to address issues associated with incompressibility as well as to increase the accuracy in the Natural Element Method (NEM). The NEM exhibits attractive features such as interpolant shape functions or auto-adaptive domain of influence, which alleviates some of the most common difficulties in meshless methods. Nevertheless, the shape functions can only reproduce linear polynomials, and in contrast to moving least squares methods, it is not easy to define interpolations with arbitrary approximation consistency. In order to treat mechanical models involving incompressible media in the framework of mixed formulations, the associated functional approximations must satisfy the well known inf-sup, or LBB condition. The first proposed approach constructs richer NEM approximation schemes by means of bubbles associated with the topological entities of the underlying Delaunay tessellation, allowing to pass the LBB and to remove pressure oscillations in the incompressible limit. Despite of its simplicity, this approach does not construct approximation with higher order consistency. The second part of the paper deals with a discussion on the construction of second-order accurate NEM approximations. For this purpose, two techniques are investigated : (a) the enrichment in the MLS framework of the bubbles with higher-order polynomials and (b) the use of a new Hermite-NEM formulation.

Key words: Natural Element Method; Bubble functions; Mixed formulations; Incompressible media; LBB condition. 


\section{Introduction}

It is well known that the solution of mechanical problems involving incompressible media using the standard displacement-based finite element technique may yield solutions that are grossly in error [2]. The difficulty is that the computed displacement field needs to satisfy the constraint of very small volumetric strains (which become zero as the condition of total incompressibility is approached) while the pressure is of the order of the boundary tractions. The displacement approximation space is not rich enough to accommodate this constraint without a drastic reduction in the rate of convergence, also known as locking [2].

For the analysis of such problems, one solution is to use a mixed formulation in which different approximation spaces are used for the displacement and pressure fields interpolation. Although numerous mixed formulations may be developed, only those that are stable are useful in practice $[12,11]$. The solvability, stability and optimality of mixed formulations are related to a compatibility condition, the so-called LBB (or inf-sup) condition [4]. The analytical proof whether the inf-sup condition is satisfied for a specific formulation is, however, difficult, and this has spurred the use of a numerical inf-sup test $[17,4,9]$.

Accounting incompressibility in meshless methods is still an open topic. Until recently, it was stated that meshfree methods are immune to locking $[3,33]$. Furthermore, the EFG has been actually proposed for treating isochoric elastoplasticity by considering the shape functions support large enough [1]. In the context of the RKPM, a similar claim was made in the context of large deformation of nearly incompressible hyperelastic [6] and elastoplastic materials [16]. Recently, it has been reported that meshfree methods are in fact not locking-free in the incompressibility limit [10]. In a recent paper [14] this issue is clarified determining the influence of the EFG shape functions support on the locking behaviour. The main conclusion was that by increasing the shape functions support the locking can be attenuated, but never suppressed. Several attempts have been proposed to avoid locking in the context of meshfree methods. Huerta et al. [27] developed a so-called pseudo-divergence free approximation, consisting in using approximation functions that verify approximately the divergence-free constraint for a given discretization in a diffuse sense. Dolbow and Belytschko [10] have proposed a mixed displacement/pressure formulation and selective reduced integration to alleviate locking. Chen et al. note that the use of large support size is computationally expensive and, moreover, cannot remove pressure oscillations [7]. They proposed a pressure projection combined with a reduced integration to remove pressure oscillations in nearly incompressible elasticity problems.

In this paper, we focus on the treatment of incompressibility in the context of the natural element method (NEM). The NEM is a novel meshfree method. Its attractive features are: (a) interpolant character of the shape 
functions; (b) strict linearity of the shape functions over the boundaries ${ }^{\S}$; and (c) the support of the shape functions is based on the Delaunay spheres of the surrounding nodes, and automatically adapts to the local nodal density. The properties (a) and (b) allow direct enforcement of the essential boundary conditions and guarantee conforming approximations in presence of interfaces [29]. Property (c) allows simple refinement strategies [31]. However, unlike the moving least square techniques, it is not possible to directly enrich the basis in order to improve the reproducing conditions (approximation consistency). In the context of incompressible media, Sukumar was the first to propose a mixed NEM interpolation in [26] using constant piecewise shape functions for the pressure approximation, and the standard NEM for the approximation of the displacements. In [13], González et al. proposed an enrichment of the NEM in the context of the partition of unity paradigm [18] to construct richer approximations, in order to verify the inf-sup condition. In [28], Chen et al. proposed to use a stabilized nodal integration to avoid locking in near-incompressible elastostatics. We propose in this paper a new approach in the context of the natural element method allowing to define stable mixed formulations for treating mechanical models involving incompressible media. In the proposed technique, additional degrees of freedom, associated with some topological entities of the underlying Delaunay tessellation, i.e. edges, triangles and tetrahedrons, are introduced. The associated bubble shape functions are computed from the product of the NEM shape functions related to the generating nodes of the entity. A Hermite-NEM approximation is also proposed, through a discussion dealing with the construction of higher-order NEM approximations.

\section{Review of the Natural Element Method}

\subsection{Natural Neighbor Interpolation}

We briefly touch upon the foundation of Sibson's natural neighbor coordinates (shape functions) that are used in the natural element method. For a more in-depth discussion on the Sibson interpolant and its application for solving second-order partial differential equations, the interested reader can refer to Sambridge and Braun [23], and Sukumar et al. [25]. The NEM interpolant is constructed on the basis of the Voronoi diagram. The Delaunay tessellation is the topological dual of the Voronoi diagram.

Consider a set of nodes $S=\left\{n_{1}, n_{2}, \ldots, n_{N}\right\}$ in $\Re^{\text {dim }}$. The Voronoi diagram is the subdivision of $\Re^{d i m}$ into regions $T_{i}$ (Voronoi cells) defined by

$$
T_{i}=\left\{\mathbf{x} \in \Re^{d i m}: d\left(\mathbf{x}, \mathbf{x}_{i}\right)<d\left(\mathbf{x}, \mathbf{x}_{j}\right), \forall j \neq i\right\}, \quad \forall i
$$

\footnotetext{
$\S$ This property is restricted to convex boundaries [25]. However, some techniques have been provided to extend it to non-convex boundaries $[30,8]$
} 


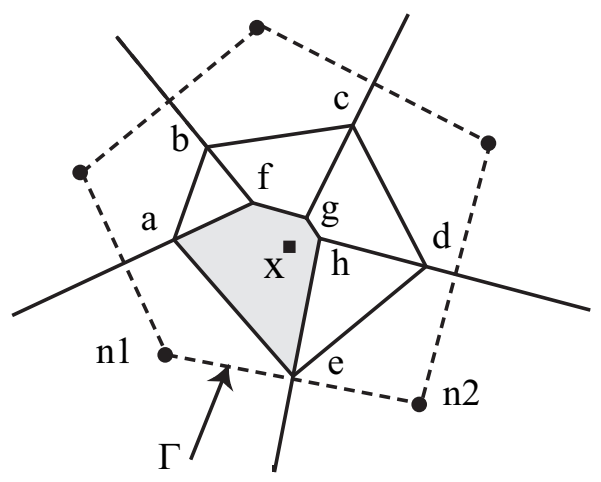

(a)

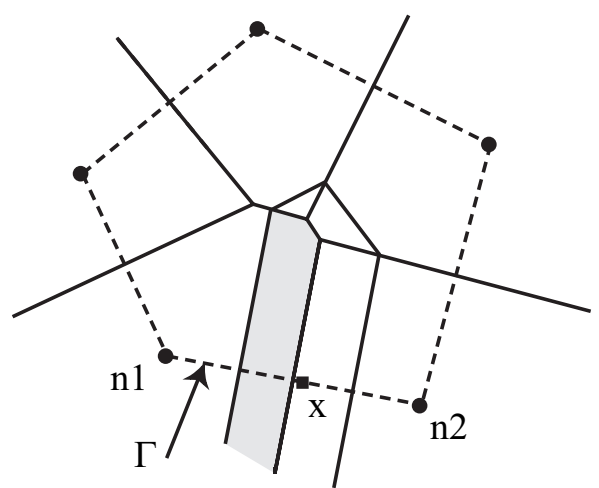

(b)

Figure 1. Construction of the Sibson shape functions.

The Sibson coordinates of $\mathbf{x}$ with respect to a natural neighbor $n_{i}$ (see Fig. 1a) is defined as the ratio of the overlap area (volume in 3D) of their Voronoi cells to the total area (volume in $3 \mathrm{D}$ ) of the Voronoi cell related to point $\mathbf{x}$. If we consider the $2 \mathrm{D}$ example depicted in figure $1(\mathrm{a})$, we have:

$$
\phi_{1}(\mathbf{x})=\frac{\text { Area }(\text { afghe })}{\text { Area }(\text { abcde })}
$$

If the point $\mathbf{x}$ coincides with the node $n_{i}$, i.e. $\left(\mathbf{x}=\mathbf{x}_{i}\right), \phi_{i}\left(\mathbf{x}_{i}\right)=1$, and all other shape functions are zero, i.e. $\phi_{j}\left(\mathbf{x}_{i}\right)=\delta_{i j}\left(\delta_{i j}\right.$ being the Kronecker delta). The properties of positivity, interpolation, and partition of unity are then verified [25]:

$$
\left\{\begin{array}{l}
0 \leq \phi_{i}(\mathbf{x}) \leq 1 \\
\phi_{i}\left(\mathbf{x}_{j}\right)=\delta_{i j} \\
\sum_{i=1}^{n} \phi_{i}(\mathbf{x})=1 .
\end{array}\right.
$$

The natural neighbour shape functions also satisfy the local coordinate property [24], namely:

$$
\mathbf{x}=\sum_{i=1}^{n} \phi_{i}(\mathbf{x}) \mathbf{x}_{i}
$$

which combined with Eq. (2.3), implies that the natural neighbour interpolant spans the space of linear polynomials (linear completeness).

Sibson natural neighbour shape functions are $C^{1}$ at any point except at the nodes, where they are only $C^{0}$. The $C^{1}$ continuity everywhere can be obtained by using special classes of natural neighbour shape functions [15].

The support (domain of influence) of a shape function $\phi_{i}$ is the union of the Delaunay spheres (circumscribing the Delaunay tetrahedrons) containing 
the node $n_{i}$. This support is thus not radial and automatically adapts to the relative position of $n_{i}$ and its neighbours, whether is the density or the regularity of the nodal distribution.

Another important property of this interpolant is its strict linearity over the boundary of convex domains. The proof can be found in Sukumar et al. [25]. An illustration is depicted in Fig. 1 (b): as the areas associated to points on the boundary become infinite, the contribution of internal nodes vanish in the limit when the point approaches the convex boundary, and the shape functions associated with nodes $n_{1}$ and $n_{2}$ become linear on the segment $\left(n_{1}-n_{2}\right)$. This is not true in the case of non convex boundaries, and an appropriate treatment must be introduced to preserve this property in nonconvex domains $[30,8]$. Essential boundary conditions can thus be enforced directly, as in the finite element method. This property also guarantees strict continuity of the approximation across material interfaces [29], which is an issue in most meshfree methods.

Consider an interpolation scheme for a vector-valued function $\mathbf{u}(\mathbf{x}): \Omega \subset$ $\Re^{2} \rightarrow \Re$, in the form

$$
\mathbf{u}^{h}(\mathbf{x})=\sum_{i=1}^{n} \phi_{i}(\mathbf{x}) \mathbf{u}_{i}
$$

where $\mathbf{u}_{i}$ are the nodal values of the field at the $n$ natural neighbour nodes, and $\phi_{i}(\mathbf{x})$ are the shape functions associated with each neighbour node. It is noted that Eq. (2.5) defines a local interpolation scheme. Thus, the trial and test functions used in the discretization of the variational formulation describing the problems treated in this paper take the form of Eq. (2.5).

One of the drawbacks of the NEM is that natural neighbour shape functions can only reproduce at best linear fields, which induces difficulties to construct mixed formulations, where the different fields must be approximated in different approximation spaces in order to avoid numerical locking (LBB condition [4]). In the next section, two new approaches are proposed to enrich the NEM approximation.

\section{Hierarchical Bubble Functions in the Natural Element Method}

Consider an open bounded domain $\Omega \in \Re^{d i m}$ with boundary $\Gamma$, dim being the space dimension. Assume that $\Omega$ is discretized by a set of nodes $S$. Let $D(S)$ be the simplicial complex associated with the Delaunay tessellation of S. A simplicial complex $K$ in $\Re^{d i m}$ is a collection of simplices (hypertetrahedra) in $\Re^{d i m}$ such that:

(i) Every face of a simplex of $K$ is in $K$;

(ii) The intersection of any two simplices of $K$ is a face of each of them [19]; 
If we denote $F_{k}$ the set of $k$-simplices $(0 \leq k \leq 3)$, in $R^{3}$ the Delaunay tessellation $D(S)$ will be defined as the simplicial complex defined by the tetrahedra in $F_{3}$, the triangles in $F_{2}$, the edges in $F_{1}$, and the vertices in $F_{0}$. We denote these collections $T(S), F(S), E(S)$ and $V(S)$, respectively.

In order to construct richer approximations, new shape functions can be associated with the different $k-$ simplices. The case $1<k<3$ is related to the concept of hierarchical methods [34]. The concept of hierarchical bubble shape functions is a very simple way to construct richer approximations. The extension to meshfree methods is not an easy matter in general, in the absence of topology related to some elements. In the natural element, the underlying Delaunay triangulation allows the use of such an approach.

The key idea is to associate new shape functions to the $k$-simplices of the Delaunay tessellation, i.e. tetrahedra $T^{\prime} \in T(S)$, triangular facets $F^{\prime} \in F(S)$ and edges connecting two nodes in the Delaunay triangulation $E^{\prime} \in E(S)$.

\section{1 b-NEM Approximation}

A k-simplex (K-S) (vertex, edge, triangular facet or tetrahedron) is generated by $K=k+1$ vertices ( $k=0,1,2$ and 3 , respectively). The bubble shape function of an entity $\chi_{j}$ generated by $K$ vertices is computed like

$$
\phi_{j}^{*}(\mathbf{x})=\prod_{p=1}^{K} \phi_{p}(\mathbf{x})
$$

where $\phi_{p}(\mathbf{x})$ is the NEM shape function (Eq. 2.2) associated with node $n_{p}$ computed at point $\mathbf{x}$.

The support (domain of influence) of a K-S generated by $K$ vertices (nodes) in $S$ is the union of the Delaunay spheres containing the $K$ nodes. It results, in $2 \mathrm{D}$ :

(i) if $\chi_{j}$ is a Delaunay triangle $(\chi \in F(S))(k=2)$, the support of $\chi_{j}$ is composed with one circle containing the 3 generating nodes of the triangle (see fig. 2 (a));

(ii) if $\chi_{j}$ is an edge of a Delaunay triangle $(\chi \in E(S))(k=1)$, the support of $\chi_{j}$ is composed of the union of two circles (see figure $2(\mathrm{~b})$ ) (if $\chi_{j} \notin \Gamma$ ), or one circle if $\chi_{j} \in \Gamma$ (see figure 2 (a)), containing the 2 generating nodes of $\chi_{j}$;

We now consider the following approximation scheme

$$
\mathbf{u}^{h}(\mathbf{x})=\sum_{i=1}^{n} \phi_{i}(\mathbf{x}) \mathbf{u}_{i}+\sum_{j=1}^{m} \phi_{j}^{*}(\mathbf{x}) \gamma_{j}
$$

where $n$ is the number of natural neighbours of point $\mathbf{x}, \phi_{i}(\mathbf{x})$ is the NEM shape function related to node $n_{i} \in S$ computed at point $\mathbf{x}, \phi_{j}^{*}(\mathbf{x})$ is the 


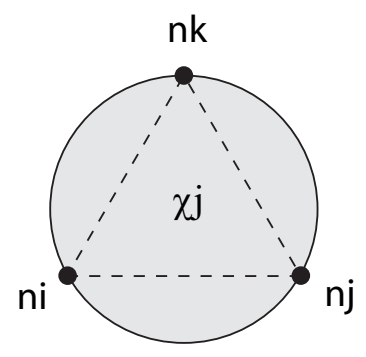

(a)

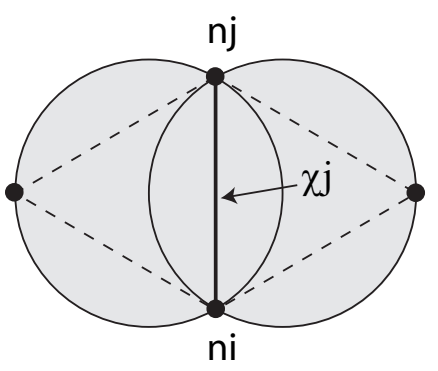

(b)

Figure 2. Supports of the bubble shape functions associated with the Delaunay k-simplex; (a) support of a Delaunay triangle $\chi_{j} n_{i}-n_{j}-n_{k}$; (b) support of a Delaunay edge $\chi_{j} n_{i}-n_{j}$.

bubble shape function defined in Eq. (3.6) associated with the $m$ influent $\mathrm{K}-\mathrm{S}$, and $\gamma_{j}$ are additional degrees of freedom.

\section{Remarks.}

(i) Different combinations can be chosen for enriching the approximation, i.e. using only bubble functions associated with the edges, with the Delaunay triangles, or both.

(ii) The evaluation of the bubble shape functions associated with the K-S is not costly as it only requires the product of available NEM shape functions computed at point $\mathbf{x}$.

(iii) Despite that the approximation scheme defined in Eq. (3.7) is richer than standard NEM approximation, it does not satisfy any reproducing property other than the linear consistency.

In [32], two variant of the b-NEM approximation have been investigated: (a) the enrichment of the approximation using one bubble function associated with each Delaunay triangle, called b1-NEM, and (b) the enrichment of the approximation using one bubble function associated with each Delaunay edge, called b2-NEM.

\section{2 b-NEM with Reproducing Properties : b-NEM ${ }^{+}$}

In this section we proceed to correct the shape functions previously constructed defining the approximation scheme (3.7) within a standard moving least squares framework, in order to evaluate the benefits provided by the higher approximation consistency. We briefly summarize the MLS procedure $[20,3]$. Let $w_{i}(\mathbf{x})$ be some weight function either associated with a standard or a bubble-NEM shape function, computed at point $\mathbf{x}$. Let the following approximation scheme defined by 


$$
\mathbf{u}^{h}(\mathbf{x})=\mathbf{p}^{T}(\mathbf{x}) \mathbf{a}(\mathbf{x})
$$

with a polynomial basis $\mathbf{p}^{T}(\mathbf{x})$, i.e. $\mathbf{p}^{T}(\mathbf{x})=[1, x, y, x y]$ and $\mathbf{p}^{T}(\mathbf{x})=$ $\left[1, x, y, x y, x^{2}, y^{2}\right]$ for a bilinear and quadratic basis, respectively, in $2 \mathrm{D}$, and $\mathbf{a}(\mathbf{x})$ a vector of unknown coefficients. In order to determine $\mathbf{a}(\mathbf{x})$, the functional $J$ defined by eq. (3.9) has to be minimized with respect to $\mathbf{a}(\mathbf{x})$ :

$$
J=\frac{1}{2} \sum_{i=1}^{n} w_{i}(\mathbf{x})\left[\mathbf{p}^{T}\left(\mathbf{x}_{i}\right) \mathbf{a}(\mathbf{x})-u_{i}\right]^{2}
$$

where $u_{i}$ are the nodal unknown associated with neighbours of point $\mathbf{x}$. The minimization of $J$ with respect to the unknown coefficient $a_{j}(\mathbf{x})$ leads to:

$$
\frac{\partial J}{\partial a_{j}(\mathbf{x})}=\sum_{k=1}^{n} a_{k}\left[\sum_{i=1}^{n} w_{i}(\mathbf{x}) p_{j}\left(\mathbf{x}_{i}\right) p_{k}\left(\mathbf{x}_{i}\right)\right]-\sum_{i=1}^{n} w_{i}(\mathbf{x}) p_{j}\left(\mathbf{x}_{i}\right) u_{i}=0
$$

which leads to the usual linear system

$$
\mathbf{A}(\mathbf{x}) \mathbf{a}(\mathbf{x})=\mathbf{B}(\mathbf{x}) \mathbf{u} .
$$

Substituting $\mathbf{a}(\mathbf{x})$ in Eq. (3.8), results in

$$
u^{h}(\mathbf{x})=\mathbf{p}^{T}(\mathbf{x}) \mathbf{A}^{-1}(\mathbf{x}) \mathbf{B}(\mathbf{x}) \mathbf{u} .
$$

By identification, the new shape functions are given by

$$
\boldsymbol{\psi}^{T}(\mathbf{x})=\mathbf{p}^{T}(\mathbf{x}) \mathbf{A}^{-1}(\mathbf{x}) \mathbf{B}(\mathbf{x}) .
$$

The reproducing b-NEM shape functions are computed by setting $w_{i}(\mathbf{x})=$ $\left\{\phi_{i}(\mathbf{x}) ; \phi_{j}^{*}(\mathbf{x})\right\}, \phi_{i}(\mathbf{x})$ and $\phi_{j}^{*}(\mathbf{x})$ being the shape functions defined in $(2.2)$ and (3.6).

In the following, $\boldsymbol{\psi}(\mathbf{x})$ is a vector containing the shape functions associated with influent nodes or $\mathrm{K}-\mathrm{S}$ at point $\mathbf{x}$.

Remark. The main difference between the reproducing-b-NEM and the bNEM without additional reproducing properties is that physical coordinates must be associated with each K-S shape function, in order to evaluate the terms $p_{j}\left(\mathbf{x}_{i}\right)$ and $p_{k}\left(\mathbf{x}_{i}\right)$ in Eq. (3.10). A simple solution is to consider the $\mathrm{K}-\mathrm{S}$ centroid coordinates.

In the following, the b1-NEM and b2-NEM schemes described in the previous section are corrected using the MLS procedure just described. In the most unfavourable case a point $\mathbf{x}$ is influenced by four shape functions in the b1-NEM (3 NEM shape functions, and 1 bubble shape function associated with the Delaunay triangle). As these weight functions are independent, the method is stable if the basis $\mathbf{p}^{T}(\mathbf{x})$ contains 4 monomials. We call b1-NEM ${ }^{+}$ 
the enrichment of the b1-NEM from $\mathbf{p}^{T}(\mathbf{x})=\{1, x, y, x y\}$. Following similar assumptions, b2-NEM ${ }^{+}$results from the enrichment of the b2-NEM using $\mathbf{p}^{T}(\mathbf{x})=\left\{1, x, y, x y, x^{2}, y^{2}\right\}$.

We have shown in [32] that essential boundary conditions can be enforced directly in all the proposed approximation schemes, as the bubble-NEM shape functions vanish over all external boundaries. For further details, see the proofs for the different schemes in the referred paper.

\subsection{Natural Element Discretization}

We consider the usual mixed variational formulation of the incompressible linear elastostatics problem where displacement trial and test functions are interpolated using the same shape functions, as the same for the pressure trial and test functions. In the following, the pressure is interpolated using the standard (Sibson) NEM shape functions, while the displacements are interpolated using the b-NEM or the b-NEM ${ }^{+}$shape functions previously defined.

\section{b-NEM Displacements Interpolation}

In the context of the b-NEM, the following approximation scheme is used for the displacements interpolation:

$$
\begin{gathered}
\mathbf{u}^{\mathbf{h}}(\mathbf{x})=\sum_{i=1}^{n} \phi_{i}(\mathbf{x}) \mathbf{u}_{i}+\sum_{j=1}^{m} \phi_{j}^{*}(\mathbf{x}) \boldsymbol{\gamma}_{j}, \quad \boldsymbol{\delta} \mathbf{u}^{\mathbf{h}}(\mathbf{x})=\sum_{i=1}^{n} \phi_{i}(\mathbf{x}) \delta \mathbf{u}_{i}+\sum_{j=1}^{m} \phi_{j}^{*}(\mathbf{x}) \boldsymbol{\delta} \boldsymbol{\gamma}_{j} \\
p^{h}(\mathbf{x})=\sum_{i=1}^{n} \phi_{i}(\mathbf{x}) p_{i}, \quad \delta p(\mathbf{x})=\sum_{i=1}^{n} \phi_{i}(\mathbf{x}) \delta p_{i}
\end{gathered}
$$

where $\phi_{i}(\mathbf{x})$ is the usual (Sibson) NEM shape function related to node $n_{i}$ computed at point $\mathbf{x}, \phi_{j}^{*}(\mathbf{x})$ is the bubble shape function associated with the $\mathrm{K}-\mathrm{S} \chi_{j}$, being $\gamma_{j}$ the degree of freedom associated with $\chi_{j}, n$ the number of neighbour nodes related to point $\mathbf{x}$ and $m$ the number of influent K-S at point $\mathbf{x}$ (number of K-S shape functions whose support contains $\mathbf{x}$ ).

\section{b-NEM+ ${ }^{+}$Interpolation}

In the context of the b-NEM ${ }^{+}$, the following approximation scheme is used for the displacements interpolation:

$$
\begin{gathered}
\mathbf{u}^{h}(\mathbf{x})=\sum_{i=1}^{n+m} \psi_{i}(\mathbf{x}) \mathbf{u}_{i}, \quad \delta u^{h}(\mathbf{x})=\sum_{i=1}^{n+m} \psi_{i}(\mathbf{x}) \delta \mathbf{u}_{i} \\
p^{h}(\mathbf{x})=\sum_{i=1}^{n} \phi_{i}(\mathbf{x}) p_{i}, \quad \delta p^{h}(\mathbf{x})=\sum_{i=1}^{n} \phi_{i}(\mathbf{x}) \delta p_{i}
\end{gathered}
$$


where $\psi_{i}(\mathbf{x})$ are the corrected shape functions computed at point $\mathbf{x}$ using the MLS technique described in section 3.2, $n+m$ the number of influent shape functions, including nodes and K-S shape functions, $\mathbf{u}_{i}$ contains both displacements associated with the nodes, and nodes that have been associated to the K-S shape functions in the MLS procedure.

\subsection{Numerical Test for the inf-sup Condition}

In order to perform the inf-sup test a sequence of successive refined meshes is considered (uniform distributions). The test is defined in details in [9]. The objective is to monitor the inf-sup values, $\beta_{\text {min }}$ (minimum eigenvalue related to the discrete LBB condition [9]), when $h$ decreases. If $\log \left(\beta_{\min }\right)$ decreases with $\log (h)$ the approximation scheme does not pass the LBB numerical test, which requires that $\log \left(\beta_{\text {min }}\right)$ remains bounded by a positive constant when $\log (h)$ decreases.

Figure 3 shows numerical test comparing some mixed NEM approximation schemes, i.e.: b-NEM/NEM, NEM/Thiessen [26] (NEM approximation for the displacements and constant pressure within each Voronoi cell), and the P1/P0 and P2/P1 mixed FEM approximation schemes. The FEM computations are carried out using directly the Delaunay triangles. As claimed in other previous works [13], the mixed NEM/Thiessen approximation scheme does not pass the numerical inf/sup test. The mixed FEM P1/P0 also violates the LBB condition [9]. All the bubble-NEM schemes are clearly LBB compliant, being the results similar to the ones computed by using the P2/P1 FEM, which satisfy the LBB condition.

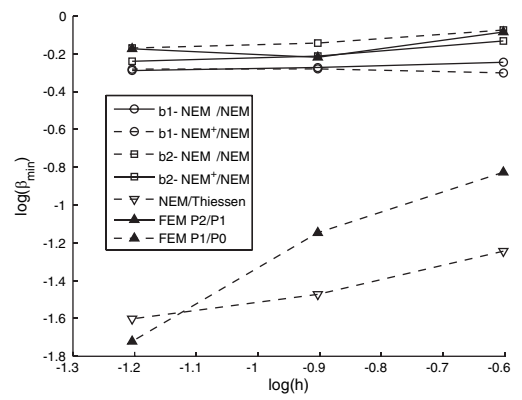

Figure 3. inf-sup numerical test.

More examples in the context of incompressible elasticity can be found in [32], in which we have shown that the b-NEM allows to remove pressure oscillations in the incompressible limit. As noted previously, the NEM shape functions only possess linear completeness [24]. In [32], we have noticed that the enrichment of bubble in the context of MLS does not seem to increase the 
convergence rate with standard integration despite the proved increase in the approximation consistency, probably due to the highly oscillating character of the obtained shape functions. In the following, a different technique is analyzed with respect to that limitation.

\section{Hermite-Natural Element Formulation}

\subsection{Hermite-NEM Approximation}

In this section, quadratic approximation consistency is achieved through a diffuse Hermite-NEM interpolation [22], by using natural neighbour weights in the moving least square approximation. Compared to standard moving least square method, the minimization is performed both with respect to the primary variable, and the diffuse spatial derivatives. For this purpose, we consider an interpolation scheme in the form

$$
u^{h}(\mathbf{x})=\sum_{i=1}^{n} \psi_{i}(\mathbf{x}) u_{i}+\sum_{i=1}^{n} \psi_{i}^{x}(\mathbf{x}) \frac{\partial u_{i}}{\partial x}+\sum_{i=1}^{n} \psi_{i}^{y}(\mathbf{x}) \frac{\partial u_{i}}{\partial y}
$$

where $\psi_{i}(\mathbf{x})$ are the shape function associated with the unknown variable $u_{i}$, $\psi_{i}^{x}(\mathbf{x})$ and $\psi_{i}^{y}(\mathbf{x})$ are the shape function associated with the spatial diffuse derivative of $u_{i}$ with respect to $x$ and $y$, respectively. In the above framework, $u_{i}, \frac{\partial u_{i}}{\partial x}$ and $\frac{\partial u_{i}}{\partial y}$ are unknown (degrees of freedom). In order to construct the shape functions, we consider the minimization of the functional

$$
u^{h}(\mathbf{x})=\mathbf{p}(\mathbf{x})^{T} \mathbf{a}
$$

where $\mathbf{p}(\mathbf{x})$ is a polynomial basis, i.e. $\mathbf{p}(\mathbf{x})=\left\{1, x, y, x y, x^{2}, y^{2}\right\}$ and $\mathbf{a}$ is a vector of unknown coefficients. In order to determine $\mathbf{a}$, we consider the functional

$$
\begin{aligned}
J=\frac{1}{2} \sum_{i=1}^{n} w_{i}(\mathbf{x})\{ & {\left[\mathbf{p}^{T}(\mathbf{x}) \mathbf{a}-u_{i}\right]^{2}+\alpha\left[\frac{\partial \mathbf{p}^{T}}{\partial x}(\mathbf{x}) \mathbf{a}-\frac{\partial u_{i}}{\partial x}\right]^{2}+} \\
& \left.+\alpha\left[\frac{\partial \mathbf{p}^{T}}{\partial y}(\mathbf{x}) \mathbf{a}-\frac{\partial u_{i}}{\partial y}\right]^{2}\right\}
\end{aligned}
$$

where $n$ is the number of natural neighbours of point $x, w_{i}(\mathbf{x})$ are the natural neighbour shape functions computed at point $x, \frac{\partial \mathbf{p}^{T}}{\partial x}(\mathbf{x})$ and $\frac{\partial \mathbf{p}^{T}}{\partial y}(\mathbf{x})$ represent the derivative of the basis $\mathbf{p}(\mathbf{x})$ with respect to $x$ and $y$, respectively. $\alpha$ is a dimensional parameter which is fixed to 1 in our simulations. Minimizing $J$ with respect to a leads to the following system of equations:

$$
\mathbf{A a}(\mathbf{x})=\mathbf{B q}
$$

with $\mathbf{q}=\left\{u_{1}, \frac{\partial u_{1}}{\partial x}, \frac{\partial u_{1}}{\partial y}, u_{2}, \frac{\partial u_{2}}{\partial x}, \frac{\partial u_{2}}{\partial y}, \ldots, u_{N}, \frac{\partial u_{N}}{\partial x}, \frac{\partial u_{N}}{\partial y}\right\}$. 
Derivatives of the shape functions are obtained through a standard procedure [3], involving the derivative of the weight functions $w_{i}(\mathbf{x})$. Closed form of Sibson shape functions derivatives can be found in [21]. The obtained shape functions are depicted in figure 4.
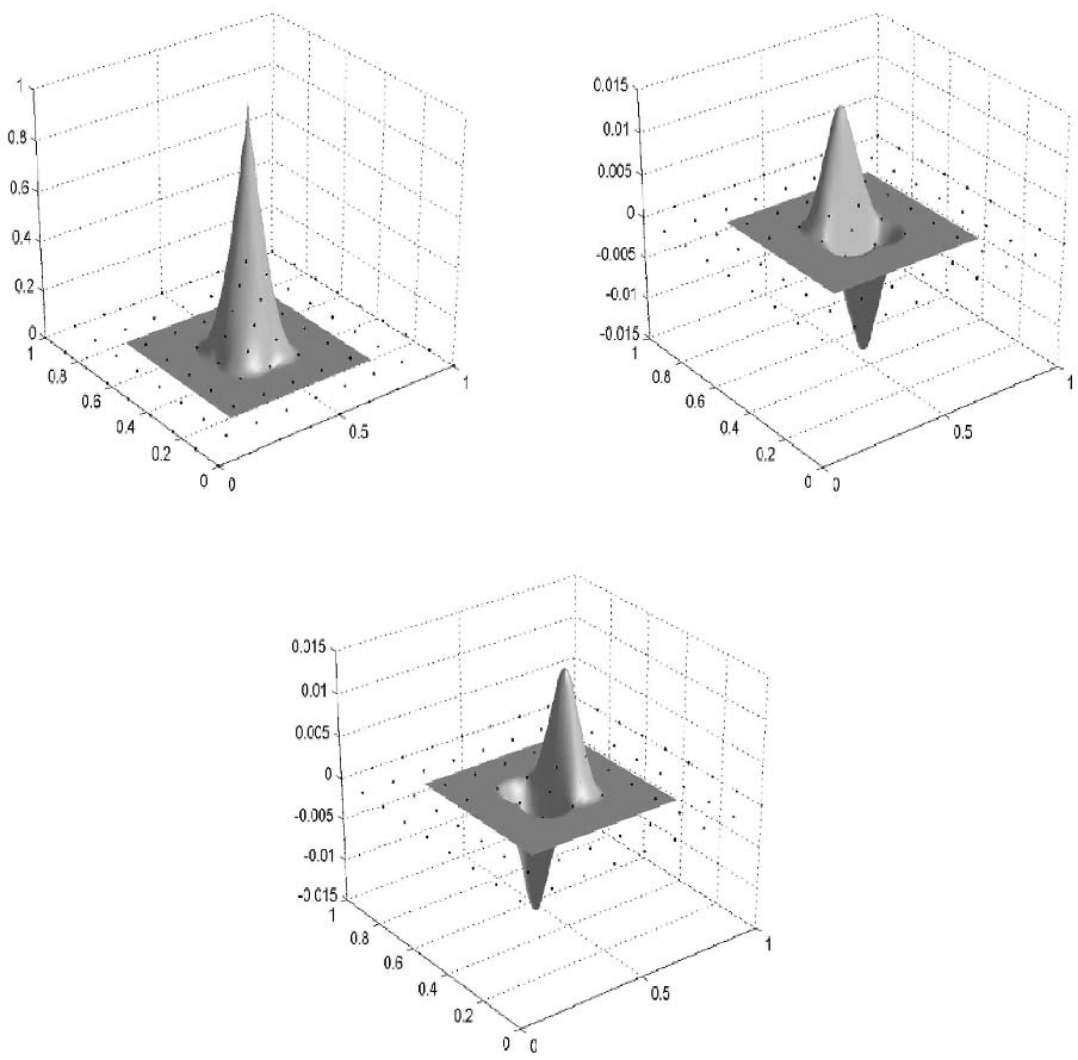

Figure 4. Hermite natural neighbour shape functions.

This approach provides smoother shape functions than the ones obtained in the context of enriched bubbles computed in the MLS framework. Then we assume that for a given number of integration points, the Hermite-NEM scheme will lead to more accurate results than the MLS-bubble NEM. Nevertheless, as in the MLS-bubble NEM there are no issues associated with the boundary conditions, it is not so obvious in the context of Hermite-NEM. According to Eq. (4.20), the new degrees of freedom associated with the derivatives can be interpreted like pseudo-derivatives which do not coincide with the real derivatives. Thus, imposition of essential boundary conditions becomes 
delicate. Nevertheless, in order to investigate the accuracy of the technique without being polluted by this issue, we consider in next section a Poisson's problem whose solution and its derivatives on the boundary vanish.

\subsection{Numerical Example Involving H-NEM Approximation}

The boundary value problem is defined by

$$
\left\{\begin{array}{l}
-\Delta u=f \quad \text { in } \Omega=] 0,1[\times] 0,1[ \\
u=\bar{u} \quad \text { on } \Gamma_{u} .
\end{array}\right.
$$

We consider from now on

$$
\left\{\begin{array}{l}
\bar{u}=0 \\
f=4 \pi^{2}\{2 \cos (2 \pi x) \cos (2 \pi y)-\cos (2 \pi x)-\cos (2 \pi y)\}
\end{array}\right.
$$

whose exact solution results in

$$
u^{e x}(\mathbf{x})=\{1-\cos (2 \pi x)\}\{1-\cos (2 \pi y)\}
$$

The weak form associated with Eq. (4.22) is given by:

Find $u \in H_{0}^{1}(\Omega)$ such that

$$
\int_{\Omega} \nabla u \cdot \nabla \delta u d \Omega \int_{\Gamma_{u}} f \delta u d \Gamma, \quad \forall \delta u \in H_{0}^{1}(\Omega)
$$

where $H_{0}^{1}(\Omega)$ is the usual Sobolev functional space. The Hermite-NEM interpolation just described is used to approximate the trial and test functions $\mathbf{u}$ and $\delta \mathbf{u}$, respectively, which are built with the only contribution of internal nodes.

The error in energy norm is computed according to

$$
\left\|\mathbf{u}-\mathbf{u}^{h}\right\|_{E(\Omega)}=\left(\frac{1}{2} \int_{\Omega}\left(\nabla u^{e x}-\nabla u^{h}\right)^{T}\left(\nabla u^{e x}-\nabla u^{h}\right)\right)^{1 / 2} .
$$

For the evaluation of both Eqs. (4.25) and (4.26), the Voronoi cells associated with each node are triangulated and a Gauss quadrature scheme is applied in each subtriangle, with 3,6 and 12 points. Figure 5 compares the accuracy of the Hermite-NEM (H-NEM) approximation with the standard NEM. If three Gauss points quadrature scheme is used, the accuracy of the H-NEM exceeds the accuracy of the NEM, but the difference in the convergence rate is not significant. If a fine enough quadrature scheme is applied (6 points), the H-NEM reaches, as expected, a second-order convergence rate. 


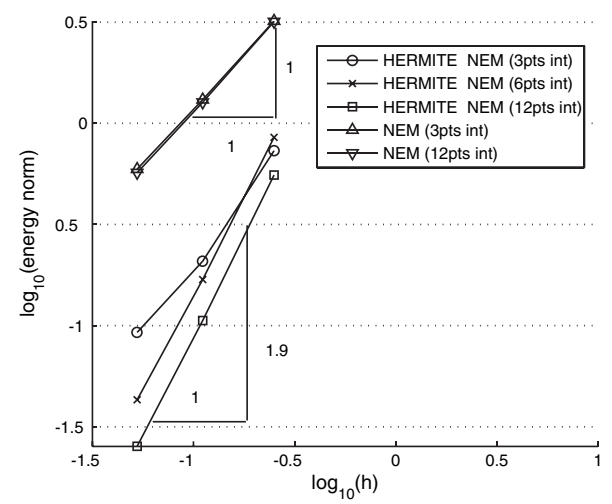

Figure 5. Convergence in energy norm for the two-dimensional Poisson's problem.

\section{Conclusion}

In this paper, new NEM approximation schemes have been proposed. On one hand, we have proposed a bubble-NEM scheme which does not increase the approximation consistency of the original NEM but allows to construct a richer approximation, which can be used in a mixed formulation to account for incompressibility. On the other hand, we have tried to construct NEM approximations with higher-order consistency. Firstly, we have proposed to enrich the bubbles in the context of the MLS scheme. Despite that this scheme has a consistency of second order in approximation, the order of convergence using standard integration remains of first order, probably due to the highly oscillatory character of the resulting shape functions. Secondly, we have proposed a Hermite-NEM scheme, in which new degrees of freedom are associated with the original nodes. The resulting shape functions are smooth enough to reach second-order convergence in absence of the difficulties related to the boundary conditions prescription.

To circumvent the difficulties related to the imposition of boundary conditions in the H-NEM framework different possibilities are presently examined: (i) the use of Lagrange multipliers; (ii) the assignment of the Hermite degrees of freedom (diffuse derivatives) to the bubble nodes instead to the original nodes, except at the bubbles located on the domain boundary where the approximation does not involve the derivatives degrees of freedom. This approach should allow to enforce essential boundary conditions, but if the resulting shape functions are not smooth enough integration difficulties could subsist being the order of convergence lower than the expected one. This analysis constitutes a work in progress. 


\section{References}

1. H. Askes, R. de Borst, O. Heeres, Conditions for locking-free elastoplastic analyses in the element-free Galerkin method, Computer Methods in Applied Mechanics and Engineering 173 (1999), 99-109.

2. K.J. Bathe, Finite Element procedures Cambridge University Press, 2001.

3. T. Belytschko, Y.Y. Lu, L. Gu, Element-free Galerkin methods, International Journal for Numerical Methods in Engineering 37 (1994), 229-256.

4. F. Brezzi, M. Fortin, Mixed and Hybrid Finite Element Methods New-York, Springer-Verlag, 1991.

5. D. Chapelle, K.-J. Bathe, The inf-sup test, Comput. Struct., 48(5) (1993), 745760 .

6. J.S. Chen, C. Pan, C.T. Wu, Large deformation analysis of rubber based on a reproducing kernel particle method, Computer methods in applied mechanics and engineering, 173 (1997), 99-109.

7. J.S. Chen, S. Yoon, H.P. Whang, W.K. Liu, An improved reproducing kernel particle method for nearly incompressible finite elasticity solids. Computer methods in applied mechanics and engineering, 181 (2000), 117-145.

8. E. Cueto, M. Doblaré, L. Gracia, Imposing essential boundary conditions in the natural elements method by means of density-scaled alpha-shapes, International Journal for Numerical Methods in Engineering 49 (2000), 519-546.

9. D. Chapelle, K.-J. Bathe, The inf-sup test, Comput. Struct., 48(5) (1993), 745760 .

10. J. Dolbow, T. Belytschko, Volumetric locking in the element-free Galerkin method, International Journal for numerical methods in engineering 46 (1999), 925-942.

11. J. Donea, A. Huerta, Finite Element methods for flow problems, John Wiley and sons, Chichester, 2003.

12. V. Girault, P.-A. Raviart, Finite element methods for Navier-Stockes equations. Theory and algorithms, Berlin, Springer-Verlag, 1986.

13. D. González, E. Cueto, M. Doblaré, Volumetric locking in natural neighbour Galerkin methods, International Journal for Numerical Methods in Engineering 61 (2004) 611-632.

14. A. Huerta, S. Fernández-Méndez, Locking in the incompressible limit for the element free Galerkin method Int. J. Numer. Methods Eng. 51(11) (2001) 13611383.

15. H. Hiyoshi, K. Sugihara, Improving continuity of Voronoi-based interpolation over Delaunay spheres, Computational Geometry 22 (2002) 167-183.

16. S. Li, W. Hoa, W.K. Liu, Mesh-free simulations of shear banding in large deformation, Int. Journ. Solids. and Sruct. 37 (2000), 7183-7206.

17. D.S. Malkus, Eigenproblems associated with the discrete $L B B$ condition for the incompressible finite elements, Int. J. Eng. Sci. 10 (1981), 1299-1310.

18. J.M. Melenk, I. Babuška, The partition of unity finite element method: basic theory and applications, Computer Methods in Applied Mechanics and Engineering 4 (1996) 289-314.

19. J.R. Munkres, Elements of algebraic topology Perseus Press, 7-14, 1993.

20. B. Nayroles, G. Touzot, P. Villon, Generalizing the finite element method: diffuse approximation and diffuse elements Computational mechanics 10 (1992) 307318. 
21. B. Piper, Properties of local coordinates based on Dirichlet tessellations, Computing Suppl. 8 (1993) 227-239.

22. A. Rassineux, P. Villon, J.M. Savignat, O. Stab, Surface remeshing by local Hermite diffuse interpolation, International Journal for Numerical Methods in Engineering 49(1-2) (2000) 10-20.

23. M. Sambridge, J. Braun, M. McQueen, Geophisical parameterization and interpolation of irregular data using natural neighbors, Geophysi. J. Int. 122 (1995) 837-857.

24. R. Sibson, A vector Identity for the Dirichlet tessellations, Math. Proc. Camb. Phil. Soc. 87 (1980) 151-155.

25. N. Sukumar, B. Moran, T. Belytschko, The natural elements method in solid mechanics, International Journal for Numerical Methods in Engineering 43 1998, 839-887.

26. N. Sukumar, The natural element method in solid mechanics. Ph.D. Dissertation. Northwestern University, Evanston, Illinois, 1998.

27. Y. Vidal, P. Villon, A. Huerta, Locking in the element free Galerkin, Communications in Numerical Methods in Engineering 19(9) (2003) 725-735.

28. J., Yoo , Moran B., Chen J.-S., Stabilized conforming nodal integration in the natural-element method, International Journal for Numerical Methods in Engineering 60 (1998) 861-890.

29. J. Yvonnet, F. Chinesta, P. Lorong, D. Rynckelynck, The constrained natural element method ( $C$-NEM) for treating thermal models involving moving interfaces, International Journal of Thermal Sciences 44 (2005), 559-569.

30. J. Yvonnet, D. Ryckelynck, P. Lorong, F. Chinesta, A new extension of the natural element method for non convex and discontinuous domains : the constrained natural element method ( $C$-NEM), International Journal for Numerical Methods in Engineering 60 (2004) 1451-1474.

31. J. Yvonnet, P. Lorong, D. Ryckelynck, F. Chinesta, Simulating dynamic thermoelastoplasticity in large transformations with adaptive refinement in the natural element method : application to shear banding, International Journal of Forming Processes, 8, (2005) 346-363.

32. J. Yvonnet, P. Villon, F. Chinesta, Natural Element Approximations involving bubbles for treating incompressible media, International Journal for Numerical Methods in Engineering, in press.

33. T. Zhu, S.N. Atluri, A modified collocation method and a penalty formulation for enforcing the essential boundary conditions in the element free Galerkin method, Computational Mechanics 21 (1998) 211-222.

34. O.C. Zienkiewicz, J.P. De, S.R. Gago, D.W. Kelly, The hierarchical concept in finite element analysis, Computers and Structures, 16 (1983) 53-65. 